\title{
A case of delayed tension pneumocephalus nine years after craniectomy
}

\author{
Ying-Tso Chen, Shu-Shong Hsu, Huang-I Hsu
}

\section{ABSTRACT}

Introduction: Pneumocephalus is a collection of air in the cranial cavity. It appears commonly after supratentorial craniotomies, whereas tension pneumocephalus is a rare but potential life-threatening complication of intracranial surgical procedures. Case Report: A 27-yearold female patient, who was diagnosed with right cerebellopontine angle tumor, received twice suboccipital craniectomies for tumor removal. Postoperatively, she had subclinical pneumocephalus, which progressed into tension pneumocephalus after nine years. Conclusion: Tension pneumocephalus may develop in a delayed manner. Therefore, close follow-up, early diagnosis and management of recurrent pneumocephalus are crucial to prevent morbidity and mortality.

Keywords: Craniectomy, Delayed, Pneumocephalus, Tension pneumocephalus

\section{How to cite this article}

Chen Y, Hsu S, Hsu H. A case of delayed tension pneumocephalus nine years after craniectomy. Int J Case Rep Images 2018;9:100917Zo1YC2018.

Ying-Tso Chen ${ }^{1}$, Shu-Shong Hsu ${ }^{2,3}$, Huang-I Hsu ${ }^{1}$

Affiliations: 'Division of Neurosurgery, Department of Surgery, Kaohsiung Veterans General Hospital, Kaohsiung, Taiwan, ROC; ${ }^{2}$ Department of Surgery, Kaohsiung Veterans General Hospital, Kaohsiung, Taiwan, ROC; ${ }^{3}$ Department of Surgery, National Defense Medical Center, Taiwan, ROC.

Corresponding Author: Shu-Shong Hsu, Department of Surgery, Kaohsiung Veterans General Hospital, No.386, Dazhong 1st Road., Zuoying Dist., Kaohsiung City 81362, Taiwan; Email: sshsu59@yahoo.com

Received: 11 April 2018 Accepted: 24 May 2018

Published: 29 May 2018
Article ID: 100917Z01YC2018

$* * * * * * * * *$

doi: 10.5348/100917Zo1YC2018CR

\section{INTRODUCTION}

Pneumocephalus refers to the collection of air in the cranium, and it occurs due to a traumatic injury or the iatrogenic violation of the dura [1]. When accumulated air exerts a mass effect on the adjacent brain parenchyma, tension physiology may develop and lead to rapid neurological deterioration or death. Although intracranial gas is commonly present for a few weeks after craniotomy [2], the delayed recurrence of pneumocephalus is not normal and may be fatal. Herein we present a case of delayed tension pneumocephalus, which developed nine years after a suboccipital craniectomy.

\section{CASE REPORT}

A 27-year-old woman, at 34 weeks pregnancy, presented with a 1-month history of progressive unsteady gait. One week before her admission, she had also experienced blurred vision, intermittent headache, nausea and vomiting. Upon examination, she presented with horizontal diplopia and difficulty in tandem gait. In addition, she had experienced hearing loss in her right ear over the prior 10 years. Magnetic resonance imaging (MRI) of her brain performed on November 9, $2007 \mathrm{had}$ revealed an extraaxial tumor, approximately $5.2 \times 5.1 \times 4.2$ $\mathrm{cm}$ in size, with heterogeneous signal intensity over the right cerebellopontine angle region (Figure 1).

She underwent cesarean section at 34 weeks and 4 days of pregnancy. However, she was drowsy postoperatively. Obstructive hydrocephalus was considered, and she received emergent external ventricular drainage on November 13, 2007. One week later, right suboccipital craniectomy for tumor removal was performed. Pathological results confirmed it to be schwannoma. After two weeks, the patient received ventriculoperitoneal 


\section{EDORIUM Journals}

www.ijcasereportsandimages.com

shunt for persistent hydrocephalus. The patient's neurological status improved, apart from the temporary postoperative presentation of partial right facial palsy, and she was discharged after seven days. Because some residual tumor was noted, the patient received adjuvant radiotherapy after the surgery.

One year later, the patient's progressive unsteady gait recurred. MRI of the brain showed residual tumor with progression. She underwent a second surgery for tumor removal in December 2008. Although residual tumor was still present, the patient was free of symptoms after the surgery (Figure 2A). A follow-up brain MRI in May 2009 revealed a newly developed pneumatocele (Figure 2B). However, the patient remained both clinically and radiographically stable (Figure $2 \mathrm{C}$ ) without evidence of cerebrospinal fluid (CSF) leakage or signs of infection during the subsequent 9-year follow-up.

In January 2018, the patient began to suffer from frequent dizziness and progressive unsteady gait. One monthlater, shealso experienced blurred vision, headache, nausea and vomiting. Worsening pneumocephalus with a focal bony defect over the right mastoid process was observed in a computed tomography (CT) scan (Figure 3). Tension pneumocephalus was impressed, and the patient underwent surgical repair of the skull bone defect and dura tear. A multilayer reconstruction of the osteodural defect was performed. We used local fat and muscle, and collagen matrix to fill the mastoid sinus, and then applied fibrin glue to seal the defect. Postoperatively, the patient achieved full clinical recovery.

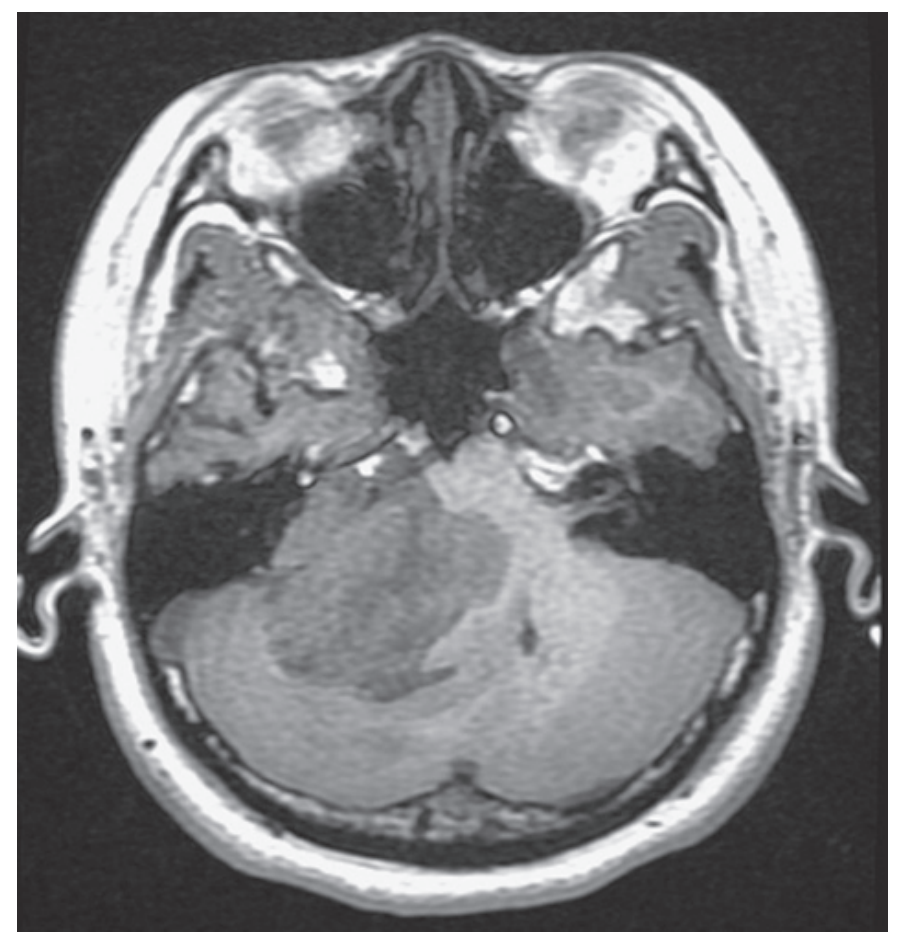

Figure 1: Preoperative brain MRI (T1 axial): large extraaxial inhomogeneous signal intensity tumor over the right cerebellopontine angle.
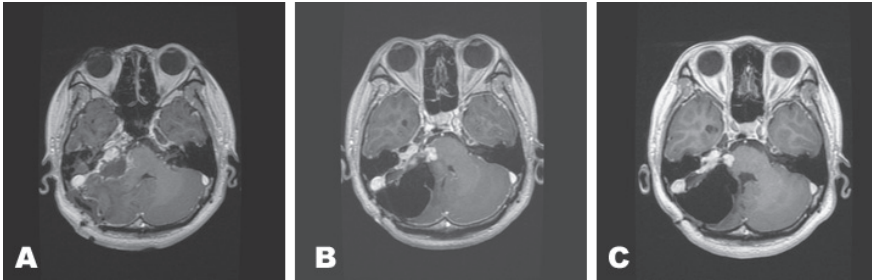

Figure 2: (A) Brain MRI (T1 with contrast) after the second surgery showed residual tumor; (B) Newly developed pneumatocele was revealed in a brain MRI 1 year after the second surgery; (C) Brain MRI eight years later showed stable pneumocephalus.

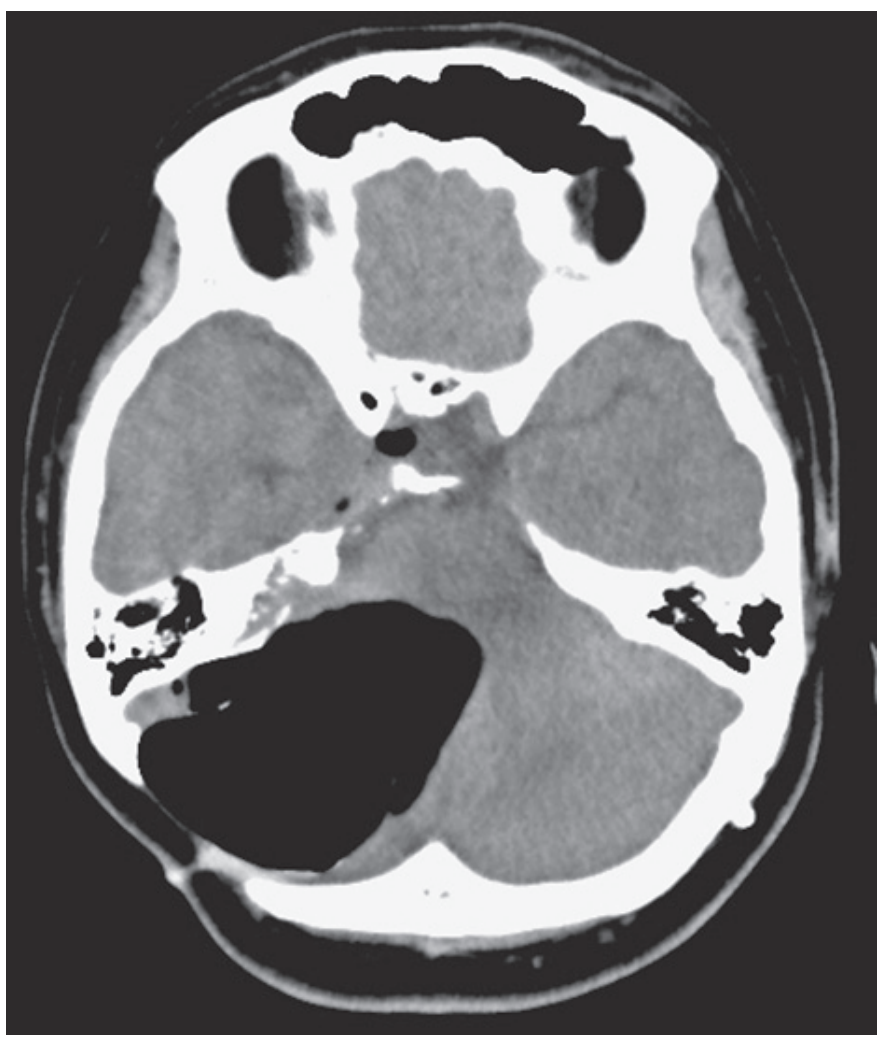

Figure 3: Brain CT depicted a larger size of pneumatocele to compress cerebellar verimis and left cerebellar hemisphere, and a focal bone defect suspected over the posterior aspect of right mastoid process.

\section{DISCUSSION}

Pneumocephalus is usually observed after neurosurgical procedures, particularly those involving the intracranial space, sinuses, orbits, or nasal cavities. Entrapment of intracranial air can be acute ( $<72$ hours) or delayed ( $\geq 72$ hours) [3]. Most intracranial accumulation of air is asymptomatic and spontaneously absorbed within 3-4 weeks [2]. Tension pneumocephalus occurs when intracranial air continues to build-up, leading to a mass effect or herniation syndrome.

Perioperative tension pneumocephalus was first described in 1962. Most previous case reports have described craniotomies involving the posterior fossa or the cervical spine. Although pneumocephalus 


\section{EDORIUM Journals}

appears after supratentorial craniotomy in almost all cases, tension pneumocephalus is observed after only $0.1 \%-0.2 \%$ of craniotomies [4]. Two main mechanisms underlying the development of pneumocephalus have been proposed. One is the ball-valve effect, where air enters through the dura defect when an increase in nasopharyngeal pressure, such as from a sneeze or cough, forces air input but not output. Another theory is the inverted soda bottle effect, which posits that when CSF lost in excess, negative pressure increases and causes air to rush in $[5,6]$. Additionally, an unusual mechanism may be the production of gas in situ due to infection by gas-producing organisms [7]. Factors that have been implicated in the pathogenesis, include nitrous oxide anesthesia, surgical position of the patient, a functional ventriculoperitoneal shunt, barotrauma, intraoperative mannitol administration, and radiotherapy $[8,9]$.

Tension pneumocephalus may manifest with a wide variety of clinical presentations, such as headache, dizziness, visual disturbance, agitation, delirium, reflex abnormalities, focal neurologic deficits, deteriorating consciousness and even cardiac arrest. Appropriate treatment for pneumocephalus varies based on severity. Most cases can be resolved with conservative treatments, including elevation of the head and avoidance of the Valsalva maneuver, as well as administration of osmotic diuretics, analgesics, and antipyretics. Studies have suggested that administration of $100 \%$ oxygen may be helpful for reabsorption of pneumocephalus $[10,11]$. However, tension pneumocephalus is considered a surgical emergency. Treatment options include the placement of a burr hole, needle aspiration, placement of a subdural or external ventricular drain, and craniotomy for decompression and repair of the dura defect.

Pneumocephalus in our patient was incidentally discovered during a regular follow-up visit one year after her surgery. Delayed onset of tension pneumocephalus occurred nine year later. Intraoperative findings showed small defect of the dura and mastoid sinus with absorption of the original muscle flap. In addition, the persistent presence of intracranial gas over years of follow-up may indicate a constant CSF fistula. The patient's delayed neurological decline was probably related to her craniectomy defect which affords space for pneumocephalus to collect. When the balance of intracranial air pressure was disturbed, continued air entrapment may have caused the mass effect, resulting in rapid neurologic deterioration. Therefore, similar cases should receive early management.

\section{CONCLUSION}

Tension pneumocephalus is a neurosurgical emergency. When the recurrent presence of intracranial air is observed, either conservative or surgical treatment is necessary to prevent progression to tension pneumocephalus.

\section{REFERENCES}

1. Komolafe EO. Tension Pneumocephalus - a rare but treatable cause of rapid neurological deterioration in traumatic brain injury: A case report. Afr J Med Sci 2010;29:88-91.

2. Reasoner DK, Todd MM, Scamman FL, Warner DS. The incidence of pneumocephalus after supratentorial craniotomy. Observations on the disappearance of intracranial air. Anesthesiology 1994 May;80(5):1008-12.

3. Pillai P, Sharma R, MacKenzie L, et al. Traumatic tension pneumocephalus: Two cases and comprehensive review of literature. OPUS 12 Scientist 2010;4(1):6-11.

4. Aydoseli A, Akcakaya MO, Aras Y, Boyali O, Unal OF. Emergency management of an acute tension pneumocephalus following ventriculoperitoneal shunt surgery for normal pressure hydrocephalus. Turk Neurosurg 2013;23(4):564-7.

5. Walker FO, Vern BA. The mechanism of pneumocephalus formation in patients with CSF fistulas. J Neurol Neurosurg Psychiatry 1986 Feb;49(2):203-5.

6. Inci S, Cirak B, Bertan V. An unusual fatal complication of low basilar trunk aneurysm surgery: Isolated prepontine tension pneumocephalus. Surg Neurol 1999 Nov;52(5):485-9.

7. Parmar MS. Pneumocephalus associated with Bacteroides fragilis meningitis. J Postgrad Med 2004 Oct-Dec;50(4):272-3.

8. Satapathy GC, Dash HH. Tension pneumocephalus after neurosurgery in the supine position. $\mathrm{Br} \mathrm{J}$ Anaesth 2000 Jan;84(1):115-7.

9. Jimenez-Jimenez E, Martí SS, Villas MV. Tension pneumocephalus related to radiotherapy for nasopharyngeal carcinoma. Case Rep Oncol Med 2014;2014:327380.

10. Gore PA, Maan H, Chang S, Pitt AM, Spetzler RF, Nakaji P. Normobaric oxygen therapy strategies in the treatment of postcraniotomy pneumocephalus. J Neurosurg 2008 May;108(5):926-9.

11. Paiva WS, de Andrade AF, Figueiredo EG, Amorim RL, Prudente M, Teixeira MJ. Effects of hyperbaric oxygenation therapy on symptomatic pneumocephalus. Ther Clin Risk Manag 2014 Oct 6;10:769-73.

$* * * * * * * * *$

\section{Author Contributions}

Ying-Tso Chen - Substantial contributions to conception and design, Acquisition of data, Analysis and interpretation of data, Drafting the article, Revising it critically for important intellectual content, Final approval of the version to be published

Shu-Shong Hsu - Substantial contributions to conception and design, Acquisition of data, Analysis and interpretation of data, Drafting the article, Revising it critically for important intellectual content, Final approval of the version to be published 
www.ijcasereportsandimages.com

Huang-I Hsu - Substantial contributions to conception and design, Acquisition of data, Analysis and interpretation of data, Drafting the article, Revising it critically for important intellectual content, Final approval of the version to be published

\section{Guarantor of Submission}

The corresponding author is the guarantor of submission.

\section{Source of Support}

None

\section{Consent Statement}

Written informed consent was obtained from the patient for publication of this case report.

\section{Conflict of Interest}

Authors declare no conflict of interest.

\section{Copyright}

(C) 2018 Ying-Tso Chen et al. This article is distributed under the terms of Creative Commons Attribution License which permits unrestricted use, distribution and reproduction in any medium provided the original author(s) and original publisher are properly credited. Please see the copyright policy on the journal website for more information.
Access full text article on other devices

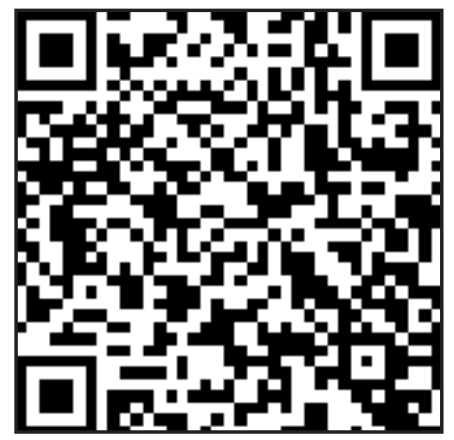

Access PDF of article on other devices

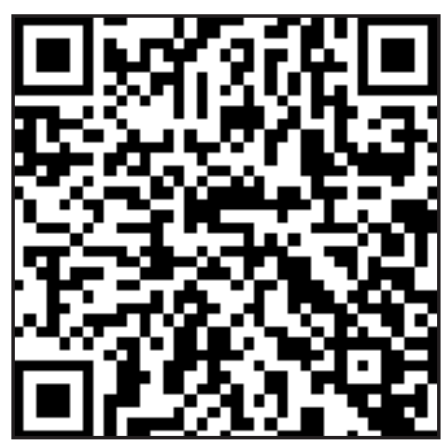

